\title{
DNA Methylation of Alu Repeats in Complicated Urinary Tract Infection in Children
}

\author{
Kalaivani Sekar ${ }^{1}$, Sriram Krishnamurthy ${ }^{1 *}$, Jharna Mandal ${ }^{2}$ and Medha Rajappa ${ }^{3}$ \\ ${ }^{1}$ Department of Pediatrics, ${ }^{2}$ Department of Microbiology, \\ ${ }^{3}$ Department of Biochemistry, Jawaharlal Institute of Postgraduate Medical Education and \\ Research (JIPMER), Puducherry, India \\ *Corresponding author
}

Keywords

Alu repeats;

Complicated

urinary tract

infection; DNA

methylation;

Epigenome;

Methylation-

specific polymerase

chain reaction

Article Info

Accepted:

20 June 2021

Available Online:

10 July 2021
Studying DNA methylation effects in Alu repetitive elements in the human genome might vary with the underlying disease state. The purpose of this study was to examine the association between Alu methylation status and complicated urinary tract infection in children. The study was conducted in the Department of Pediatrics of a referral hospital. Genomic DNA from 50 children with UTI and 50 healthy controls were isolated. The level of DNA methylation in Alu repeat was examined in these children using methylation-specific polymerase chain reaction. Highly significant difference in the percentage of Alu DNA methylation level was found between cases and controls. The mean and standard deviation of the Alu DNA methylation level between cases (26.70 118.80$)$ and controls $(38.20 \pm 18.28)(P<0.003)$. The results of this study show that Alu repeats vary significantly among cases and controls. This study indicates that interactions between Alu DNA methylation and host characteristics may determine disease progression.

\section{Introduction}

Urinary Tract Infections (UTIs) are one of the most common and severe bacterial infections in children and associated with high morbidity (1). In children, most often UTI manifests as fever of unknown origin as they often remain undiagnosed. UTIs might reflect an underlying structural defect in the kidney and urinary tract or bowel bladder dysfunction (2). Upon UTI, host immune cells undergo various defense mechanisms to trigger an appropriate immune response. The ability of the pathogen to delay or suppress immune response plays a role in the persistence of pathogens within the urinary tract (1). Susceptibility to UTI is determined by the host defense mechanism, pathogen virulence factors, underlying 
structural anomalies and also host genetic variability (3).

In humans, about $45 \%$ of the genome is composed of repetitive elements which consist of Alu repetitive elements is the most abundant and long interspersed nucleotide elements (LINE-1 elements). It is estimated that more than one-third of DNA methylation occurs in repetitive elements in the human genome (4). DNA methylation involves the addition of the methyl group at the 5'position of cytosine in $\mathrm{CpG}$ dinucleotides. This methylation of cytosine in the promoter region is associated with silencing of the gene through hypermethylation or activating genes through hypomethylation (5-7). Methylation levels of repetitive sequence such as (LINE and Alu) have been evaluated in cancer, autoimmune disease, and aging. Alu and LINE-1 hypomethylation are most commonly reported in cancer $(8,9)$.

Research suggests that pathogen-mediated DNA methylation influences the gene expression pattern contributing to disease progression. Some of the pathogen-mediated DNA methylation modifications are seen in Helicobacter pylori infection of the stomach, Schistosoma infection of the bladder, and Uropathogenic E.coli (UPEC) infection of uroepithelial cells, and neonatal sepsis (1013). Thus, methylation of repetitive elements throughout the human genome is a significant contributor to total genomic DNA methylation $(4,14)$. Presently DNA methylation is extensively studied for specificity, sensitivity, and prognostic efficacy for infections. The study is based on the hypothesis that UTI may lead to modification of the epigenome thereby increasing susceptibility to UTI. The current study aimed to investigate the methylation status of Alu repetitive element in children with complicated urinary tract infection and healthy controls by methylation-specific polymerase chain reaction (MS-PCR) method.

\section{Materials and Methods}

This study was conducted in the Department of Pediatrics of a referral hospital. The study was approved by the Institute Scientific Advisory and Human Ethics Committees. In this study, children aged 1month-18 years (either gender) with clinical symptoms consistent with complicated UTI (as defined by the revised guidelines formulated by the Indian Society of Pediatric Nephrology) were included (15). Children who had received steroids/immunosuppressants in the last 90 days or presenting $48 \mathrm{hrs}$ after initiation of antibiotic therapy or chronic kidney disease stage 4 and 5 were excluded. Children aged $1 \mathrm{~m}$ to 18 years without symptoms of complicated UTI, those without structural anomalies of the kidney and urinary tract, and any other infections were enrolled as controls. For this study, 50 children with complicated UTI and 50 healthy children were enrolled after obtaining informed signed consent from the parents and assent from children. Blood sample $(1.5 \mathrm{~mL})$ was collected in an EDTA vacutainer from the cases and controls. DNA was isolated from whole blood $(300 \mu \mathrm{L})$ using Genomic DNA Mini kit (Favorgen Biotech corp., Taiwan) as per the manufacturer's protocol. The quality of DNA was measured using Nanodrop 2000 spectrophotometer (Thermo Scientific, USA) with a ratio of 1.82.0.

Bisulfite conversion of genomic DNA was conducted using the EZ DNA MethylationGold Kit (Zymo Research Inc., USA) according to the manufacturer's protocol. Briefly, 500ng of genomic DNA from both cases and controls were used to distinguish unmethylated $\mathrm{C}$ to $\mathrm{U}$ and methylated $\mathrm{C}$ remains unchanged. The bisulfite-modified genomic DNA was suspended in $15 \mu \mathrm{L}$ of elution buffer and stored at $-20^{\circ} \mathrm{C}$. Bisulfite modified DNA was subjected to Alu methylspecific PCR (MSP). Two sets of primers 
were used to discriminate between methylated and unmethylated cases and controls. Methylated (5'-CGGATTATTTGAGGTTAG GAGTTC-3') forward and (5'-CCAAACTAA AATACAATAACGCGAT-3') reverse (203 bp product) were subject to 35 reaction cycle at $53 \mathrm{C}^{\circ}$ annealing temperatures. While unmethylated (5'-GTGGATTATTTGAGGTT AGGAGTTT-3') forward and (5' -CCAAACT AAAATACAATAACACAAT-3') reverse (204 bp product) were subject to 35 reaction cycle at $58.5 \mathrm{C}^{\circ}$ annealing temperatures (Table $1)$.

Amplification conditions were as follows: bisulfite-converted DNA $1 \mu \mathrm{l}, 10 \mathrm{pmol}$ of each forward and reverse primers $1 \mu \mathrm{l}, 7 \mu \mathrm{l}$ of nuclease-free water (Agilent Surecycler, CA, USA), and $10 \mu \mathrm{l}$ of ZymoTaq master mix were combined in a final volume of $20 \mu \mathrm{l}$ reactions.

\section{Scoring strategy of methylated samples from MS-PCR analysis}

To quantify DNA methylation levels, electrophoretic bands were scanned and their absorbance was analyzed using the Image $\mathbf{J}$ Software. The band intensity of the PCR amplified products observed in agarose gel produced in both $\mathrm{M}$ and U-specific MS-PCR represent methylation and unmethylation respectively. The intensity of the MS-PCR Products was analyzed and recorded accordingly regarding positive control run paralleled and compared with control standards i.e. $100 \%, 75 \%, 50 \%, 25 \%, 10 \%$, and $0 \%$. The control standards were obtained by combining the proportions of methylated (M) and Unmethylated (U) human control DNA (Table 2). The same methylation distribution scoring was performed to the healthy control and samples were represented according to the band intensity. To rule out any bias during the MS-PCR program stringent PCR conditions were followed and the results were interpreted according to the sample.

\section{Agarose gel Electrophoresis}

The PCR amplified product was separated in $1.5 \%$ agarose gel electrophoresis and the intensity of the products and presence/absence of both $\mathrm{M}$ and $\mathrm{U}$ MS-PCR products were captured in Image Quant LAS 500 (GE Healthcare, UK) and compared with control standards. Based on the intensity of the PCR bands the distribution of methylation level was categorized

All statistical analysis was performed in SPSS v20 and MS Excel at a 95\% confidence interval at a $5 \%$ level of significance. The distribution of categorical data on gender, disease status, type of organism, etc was expressed as frequency and percentages. Age of the enrolled children was expressed as median (Range) in both the cases and controls and compared using Mann U Whitney test.. P $<0.05$ considered as significant. Independent samples $t$-test was used to evaluate the significant differences in Alu DNA methylation between cases and controls. Percentage of Alu DNA methylation level was expressed as mean with a standard deviation between cases and controls. To check the significant differences in Alu DNA methylation and unmethylation within cases and controls Paired 't' test was carried out.

\section{Results and Discussion}

The baseline characteristics of children with complicated UTI and healthy children were depicted in Table 3. Thirty-seven of them had E.coli (74\%), and remaining were Klebsiella pneumoniae (12\%), Enterococcus (8\%), Enterobacter (4\%), Citrobacter koseri (2\%). Two sets of primer were successfully designed to discriminate between methylated and unmethylated Alu repeat elements, and the methylation level of Alu repeats was analyzed by Methyl-specific PCR in UTI cases and healthy children and the gel image was 
captured. MSP product was $203 \mathrm{bp}$ for methylated and 204 bp for unmethylated (Figure 1). Universal methylated and unmethylated human DNA standards were used as control DNA.

The image captured was used to analyze and calculate the percentage of Alu DNA methylation level in cases and controls using Image Quant software. The percentage of Alu DNA methylation in cases and controls was calculated based on the intensity of a product by comparing with control standards. The control standards were prepared with a mixture of both $\mathrm{M}$ and U DNA and subjected to MS-PCR procedure (Figure 2A).

Alu DNA methylation level was assessed between cases and controls. The comparison of the percentage of Alu DNA methylation between cases and controls showed a significant difference. The mean and standard deviation of the Alu DNA methylation level between cases and controls was $(26.70 \pm 18.80)$ and $(38.20 \pm 18.28)$ the independent samples ' $t$ ' test shows the results, p-value $(<0.003)$ (Table 4). This indicates that cases were hypomethylated when compared to controls. DNA methylation occurrence in Alu repetitive element was observed in both cases and controls. Some of the cases only methylated bands were seen in gel with the complete absence of unmethylated bands (Figure 2B).

Alu DNA methylation level was assessed among cases and controls. The percentage of Alu DNA methylation among cases and controls was shown in (Table 5). A significant difference in the percentage of Alu DNA methylation level was observed within cases, the percentage of methylation and unmethylation $(26.7 \pm 18.80)$ and $(38.4 \pm 21.76)$ the paired ' $\mathrm{t}$ ' test shows the results, $\mathrm{p}$-value $(<0.012)$ are significant. Among healthy controls, the percentage of methylation and unmethylation $(38.2 \pm 18.28)$ and $(23.5 \pm 15.16)$ $(P<0.001)$. The present study showed that the frequency of Alu DNA methylation and unmethylation within cases $(28 \%, 54 \%, 10 \%$, $8 \%)$ and controls $(12 \%, 36 \%, 44 \%, 8 \%)$ were represented in (Figure 3).

DNA methylation is a chemical change that occurs when DNA methyltransferase (DNMT) can transfer a methyl group from S-adenosylmethionine to cytosine in $\mathrm{CpG}$ dinucleotides (16). DNA methylation plays a pivotal role in gene expression, embryonic development, differentiation, chromatin structure, and genomic stability. Aberrant DNA methylation, both hypermethylation, and hypomethylation have been associated with aging, cancer, and other diseases (17-19). There are $~ 1.4$ million Alu repetitive elements and a half-million long interspersed nucleotide elements (LINE-1 elements) that are generally methylated in the human genome. Alu elements may be more affected in terms of DNA methylation than other repetitive elements due to their relatively high $\mathrm{CpG}$ density $(20,21)$.

The manifestation of DNA methylation in Alu repeats from whole blood was analyzed in UTI cases and HC. Hypomethylation of Alu repeats was observed among UTI cases. On the other hand, HC showed hypermethylation in $A l u$ repeats. Such variation could be attributed to the disease condition, state of disease progression, host genetic pattern and type of organism specific. Hypermethylation in $\mathrm{HC}$ indicates the trend of the presence of active de nova DNA methylation of Alu element than UTI cases (22). 
Table.1 Methylation-specific polymerase chain reaction primer details

\begin{tabular}{|c|c|c|c|c|c|c|}
\hline Gene & Primer & $\begin{array}{c}\text { Tm } \\
\left({ }^{\circ} \mathbf{C}\right)\end{array}$ & $\begin{array}{c}\text { CG } \\
\mathbf{\%}\end{array}$ & “'C”s & Sequence (5'-3') & bp \\
\hline Alu & F & 53 & 58.33 & 4 & CGGATTATTTGAGGTTAGGAGTTC & 20 \\
M & R & & 60.0 & 9 & CCAAACTAAAATACAATAACGCGAT & 3 \\
\hline Alu U & F & 58.5 & 60.0 & 4 & GTGGATTATTTGAGGTTAGGAGTTT & 20 \\
& R & & 68.0 & 9 & CCAAACTAAAATACAATAACACAAT & 4 \\
\hline
\end{tabular}

M: Methylation-specific primer, U: Unmethylation-specific primer, Tm: Melting temperature, F: Forward primer, R: Reverse primer, bp: Base pair in length

Table.2 Preparation of control standards

\begin{tabular}{|c|c|c|}
\hline Percentage (\%) & $\begin{array}{c}\text { Unmethylated Human DNA } \\
\text { (Negative control) } \mathbf{1 0 0 n g} / \boldsymbol{\mu l}\end{array}$ & $\begin{array}{c}\text { Methylated Human DNA } \\
\text { (Positive control) 100ng/ } \boldsymbol{\mu l}\end{array}$ \\
\hline $\mathbf{0}$ & 10.0 & 0 \\
\hline $\mathbf{1 0}$ & 9.0 & 1.0 \\
\hline $\mathbf{2 5}$ & 7.5 & 2.5 \\
\hline $\mathbf{5 0}$ & 5.0 & 5.0 \\
\hline $\mathbf{7 5}$ & 2.5 & 7.5 \\
\hline $\mathbf{1 0 0}$ & 0 & 10.0 \\
\hline
\end{tabular}

Table.3 Baseline characteristics of enrolled children:

\begin{tabular}{|c|c|c|c|}
\hline S. No & Characteristics & N=50 (cases) & N=50 (controls) \\
\hline $\mathbf{1}$ & Age (months) & $17(1,144)$ & $42(6,132)$ \\
\hline $\mathbf{2}$ & Male & $31(62 \%)$ & $30(60 \%)$ \\
\hline $\mathbf{3}$ & Causative micro-oganisms detected on urine culture (\%) & $37(74 \%)$ & - \\
& E.coli & $6(12 \%)$ & \\
& Klebsiella pneumoniae & $4(8 \%)$ & \\
& Enterococcus fecalis & $2(4 \%)$ & \\
& Enterobacter & $1(2 \%)$ & \\
\hline $\mathbf{4}$ & Citrobacter koseri & $41(82 \%)$ & \\
& Type of UTI & $7(14 \%)$ & - \\
& a) Complicated UTI & $2(4 \%)$ & \\
\hline $\mathbf{5}$ & b) Recurrent UTI & $24.28(9,69)$ & \\
& c) Breakthrough UTI & $0.72(0.3,7.68)$ & - \\
& Blood values & $61.48(6.18,151.4)$ & \\
\hline $\mathbf{6}$ & Urea (mg/dl) & $10(20 \%)$ & \\
& Creatinine (mg/dl) & $10(20 \%)$ & \\
& eGFR(ml/min/1.73m $\left.{ }^{2}\right)$ & $3(6 \%)$ & - \\
\hline $\mathbf{7}$ & CAKUT & $6(12 \%)$ & \\
\hline
\end{tabular}

CAKUT-Congenital anomalies of kidney and urinary tract; VUR-Vesicoureteral reflux; HDN-Hydronephrosis; PUV-Posterior Urethral valves; eGFR-Estimated Glomerular Filtration Rate by modified Schwartz formula All values are depicted as Median (range) or $\mathrm{n}(\%)$ 
Table.4 Comparison of Percentage of Alu DNA methylation between cases and controls

\begin{tabular}{|c|c|c|}
\hline Parameters & \% of Alu DNA methylation (Mean \pm SD) & $\boldsymbol{P}$ value \\
\hline Cases $(\mathbf{n = 5 0})$ & $26.70 \pm 18.80$ & $P<0.003$ \\
\hline $\begin{array}{c}\text { Controls } \\
(\mathbf{n = 5 0})\end{array}$ & $38.20 \pm 18.28$ & \\
\hline
\end{tabular}

Table.5 Percentage of Alu DNA methylation within cases and controls

\begin{tabular}{|c|c|c|c|}
\hline Parameters & MS-PCR & $\begin{array}{c}\text { \% of Alu DNA methylation } \\
\text { (Mean } \mathbf{\pm S D})\end{array}$ & $\boldsymbol{P}$ value \\
\hline Cases $(\mathbf{n = 5 0})$ & Methylation & $26.70 \pm 18.80$ & $<0.012$ \\
& Unmethylation & $38.40 \pm 21.77$ & \\
\hline Controls $(\mathbf{n = 5 0})$ & Methylation & $38.20 \pm 18.28$ & $<0.001$ \\
& Unmethylation & $23.50 \pm 15.16$ & \\
\hline
\end{tabular}

Fig.1 Gel electrophoresis of MSP product of Alu using 1.5\% agarose gel.

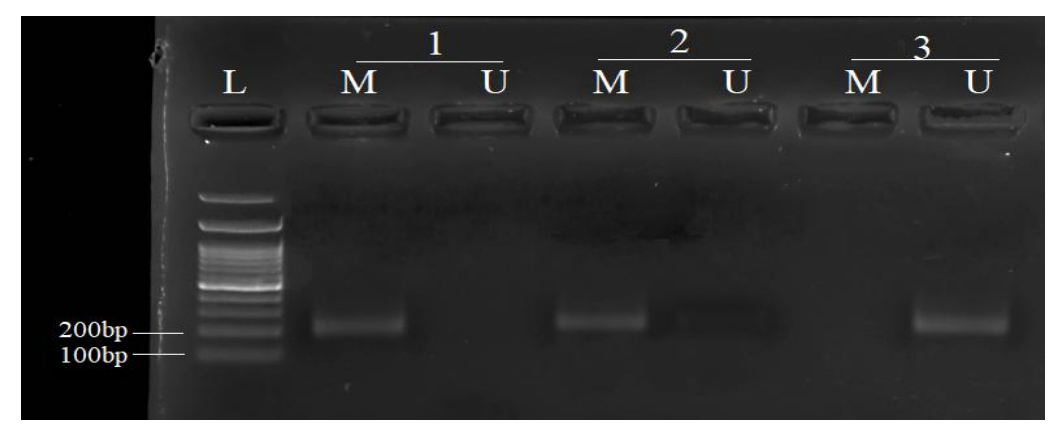

M: Product amplified with methylated-specific primer (203bp); U: Product amplified with unmethylated-specific primer (204bp)

Lane 1: Positive methylated control; Lane3: Positive unmethylated control; Lane 2: Case

Fig.2 Methylation-specific polymerase chain reaction (MS-PCR) results
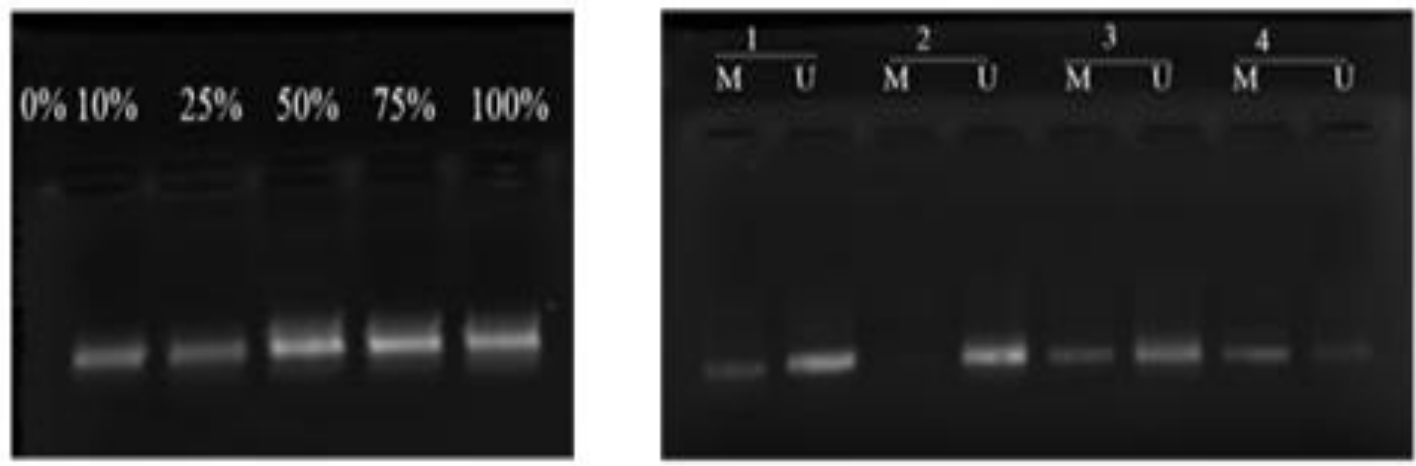

A. Gel image of $\%$ of control standards DNA

B. Methylation-specific polymerase chain reaction result of cases 
Fig.3 Frequency of Alu DNA methylation and unmethylation within cases and controls

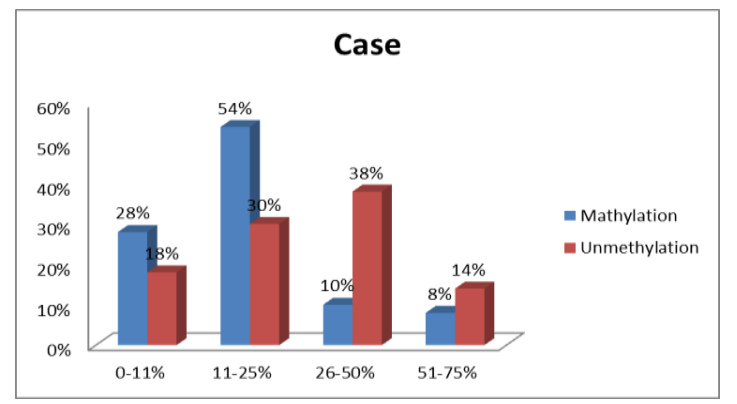

$\mathbf{M}=$ methylation frequency; $\mathbf{U}=$ unmethylation frequency

The DNA methylation variation found in cases might have been pathogen-induced as a result of the host response to infection. The difference among healthy controls indicates that the appearance of more methylation of cytosine residues in Alu DNA elements than UTI cases. The hypomethylation of Alu repeats in UTI cases indicates that it can influence the expression of inflammatory genes to aid the pathogen to prevent host immune response or expand the possibilities of appropriate immune gene expression to control UTI infection.

In most CGIs, the human genome has hypomethylation in common to retain its open chromatin state to affect the expression of neighbouring genes. On the contrary, hypermethylation was noted in repetitive elements (REs) such as LINEs, SINEs and LTRs to prevent their transcription and transposition to maintain the integrity of the genome $(23,24)$. Alu hypomethylation has been reported in several diseases but the mechanism responsible for Alu hypomethylation in UTI remains unknown. This is the first study to evaluate Alu DNA methylation in complicated UTI and offer useful insights into the pathogenesis of UTI, which may influence the expression of immune genes.

Alu hypomethylation has been reported in several diseases (132-136) but the mechanism

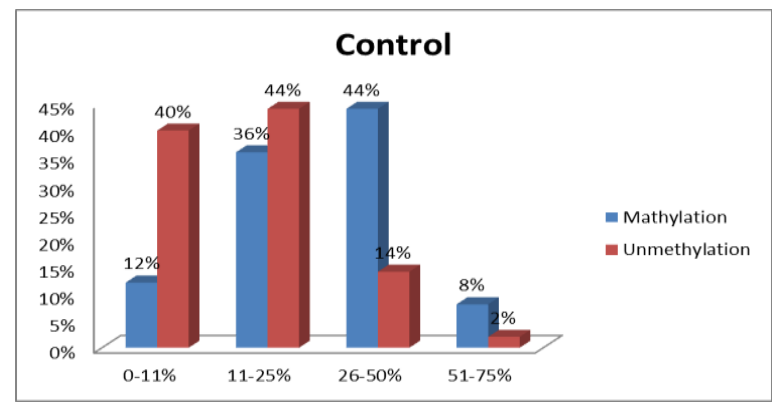

responsible for Alu hypomethylation in UTI remains unknown. This is the first study to evaluate Alu DNA methylation in complicated UTI and offer useful insights into the pathogenesis of UTI, which may influence the expression of immune genes. There are certain limitations to our study. We studied Alu DNA methylation in blood samples only (and not in urine shed uroepithelial) for logistic reasons. Children with complicated UTI often have signs of systemic toxicity, making the study of blood samples a reasonable approach for assessing Alu DNA methylation in the blood. A field of future research could be the study of DNA methylation in shed uroepithelial cells.

Our findings provide preliminary information on DNA methylation variation in Alu elements upon UTI in children. This knowledge may apply for future research.

Further research should target assessing the level of DNA methylation at genome-wide by advanced technologies. The impact of DNA methylation generates a crucial need for effective methods with high sensitivity and reliability to explore advanced diagnostic and therapeutic strategies.

\section{Acknowledgment}

This study was supported by an intramural grant from JIPMER which is gratefully acknowledged. 


\author{
Abbreviations \\ CAKUT- Congenital anomalies of kidney and \\ urinary tract \\ DNMT - DNA methyltransferase \\ HDN - Hydronephrosis \\ LINE - Long interspersed nucleotide elements \\ LTR- Long Terminal repeats \\ MS-PCR- Methylation Specific Polymerase Chain \\ reaction \\ PUV - Posterior Urethral valves \\ RE - Repetitive elements \\ SINE - Short Interspersed Elements \\ UTIs - Urinary tract infections \\ UPEC - Uropathogenic Escherichia.coli \\ VUR - Vesicoureteral reflux
}

\section{References}

1. Spencer J D, Schwaderer A L, Becknell B, Watson J, Hains DS. 2014. The innate immune response during urinary tract infection and pyelonephritis. Pediatr Nephrol. 29(7): 1139-1149.

2. Taneja N, Chatterjee S S, Singh M, Singh S, Sharma M. 2010. Paediatric urinary tract infections in a tertiary care centre from North India. Indian J Med Res. 131: 101-105.

3. Bien J, Sokolova O, Bozko P. 2012. Role of uropathogenic Escherichia coli virulence factors in development of urinary tract infection and kidney damage. Int J Nephrol. 1-15.

4. Yang A S, Estecio M R, Doshi K, Kondo Y, Tajara E H, Issa J P. 2004. A simple method for estimating global DNA methylation using bisulfite PCR of repetitive DNA elements. Nucleic Acids Res. 32: e38.

5. Jones P A, Takai D. 2001. The role of DNA methylation in mammalian epigenetics. Science. 293: 1068-1070.

6. Jones P A, Baylin S. 2002. The fundamental role of epigenetic events in cancer. Nature Rev Genet. 3: 415-428.

7. Feinberg A P, Gehrke C W, Kuo K C, Ehrlich M. 1998. Reduced genomic 5- methylcytosine content in human colonic neoplasia. Cancer Res. 48: 1159-1161.

8. Pavicic W, Emmi I, Taina N, Peltomäki P. 2012. LINE-1 hypomethylation in familial and sporadic cancer. J Mol Med. 90: 827-835.

9. Christensen B C, Houseman E A, Marsit C J, Zheng S, Wrensch M R, Wiemels J $\mathrm{L}$, et al., 2009. Aging and environmental exposures alter tissue-specific DNA methylation dependent upon $\mathrm{CpG}$ island context. PloS Genet. 5: e1000602.

10. Niwa $\mathrm{T}$, Tsukamoto $\mathrm{T}$, Toyoda $\mathrm{T}$, Mori A, Tanaka H, Maekita T, et al., 2010. Inflammatory processes triggered by Helicobacter pylori infection cause aberrant DNA methylation in gastric epithelial cells. Cancer Res 70:14301440.

11. Zhong X, Isharwal S, Naples J M, Shiff C, Veltri R W, Shao C, 2013. et al., Hypermethylation of Genes detected in urine from ghanaian adults with bladder pathology associated with Schistosoma haematobium infection. PLoS One. 8: e59089.

12. Tolg C, Sabha N, Cortese R, Panchal T, Ahsan A, Soliman A, et al., 2011. Uropathogenic E.coli infection provokes epigenetic down regulation of $\mathrm{CDKN} 2 \mathrm{~A}$ (p16INK4A) in uroepithelial cells. Invest. 91: 825-836.

13. Dhas B B, Antony H A, Bhat V, Newton B, Parija S C. 2015. Global DNA methylation in neonatal sepsis. Indian $\mathrm{J}$ Pediatr. 82: 340-344.

14. Hou L, Wang H, Sartori S, Gawron A, Lissowska J, Bollati V, et al., 2010. Blood leukocyte DNA hypomethylation and gastric cancer risk in a high-risk Polish population. Int J Cancer. 127: 1866-1874.

15. Indian Society Pediatric Nephrology, Vijayakumar M, Kanitkar M, Nammalwar BR, et al., 2011. Revised Statement on Management of Urinary Tract Infections. Indian Pediatr. 48: 709-717. 
16. Bird A. 1992. The essentials of DNA methylation. Cell 70: 5-8.

17. Issa J P. CpG-island methylation in aging and cancer. 2000. Curr Top Microbiol Immunol. 249: 101-118.

18. Richardson B. Impact of aging on DNA methylation. 2003. Ageing Res Rev. 2: 245-261.

19. Fawzi M, Al-khafaji Z M, Al-Thamir S N, Hashim H O, Mousa M J, Muslim O T. 2016. Aberrant LINE-1 Methylation upon Helicobacter pylori infection. European $\mathbf{J}$ Biomed Pharm Sci. 3: 486-492.

20. Xing J, Hedges D J, Han K, Wang H, Cordaux R, Batzer M A. 2004. Alu element mutation spectra: molecular clocks and the effect of DNA methylation. J Mol Biol. 344: 675-682.
21. Wilson A, Power B, Molloy P. 2007. DNA hypomethylation and human diseases. Bioch Biophys. 1775: 138-162.

22. Oler A, Traina-Dorge S, Derbes R S, Canella D, Cairns BR, Roy-Engel AM. 2012. Alu expression in human cell lines and their retrotranscriptional potential. Mob DNA. 3:11.

23. Xie $H$, Wang $M$, Bonaldo Mde $F$, Rajaram V, Stellpflug W, Smith C, et al., 2010. Epigenomic analysis of Alu repeats in human ependymomas. Proc Natl Acad Sci USA. 107: 6952-6957.

24. Zheng Y, Joyce B T, Liu L, Zhang Z, Kibbe W, Zhang W, Hou L. 2017. Prediction of genome-wide DNA methylation in repetitive elements. Nucleic Acids Res. 45: 8697-8711.

\section{How to cite this article:}

Kalaivani Sekar, Sriram Krishnamurthy, Jharna Mandal and Medha Rajappa. 2021. DNA Methylation of Alu Repeats in Complicated Urinary Tract Infection in Children. Int.J.Curr.Microbiol.App.Sci. 10(07): 629-637. doi: https://doi.org/10.20546/ijcmas.2021.1007.068 